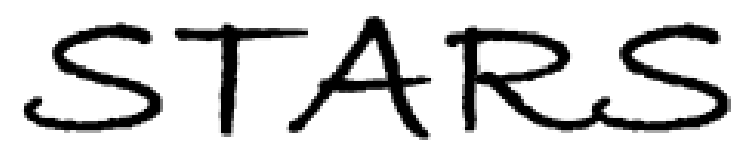

University of Central Florida

STARS

$1-1-2012$

\title{
Fixed-point attractor for chirp in nonlinear waveguide arrays
}

Darren D. Hudson

\section{J. Nathan Kutz}

Thomas R. Schibli

Qing Chao

Demetrios N. Christodoulides

University of Central Florida

See next page for additional authors

Find similar works at: https://stars.library.ucf.edu/facultybib2010

University of Central Florida Libraries http://library.ucf.edu

This Article is brought to you for free and open access by the Faculty Bibliography at STARS. It has been accepted for inclusion in Faculty Bibliography 2010 s by an authorized administrator of STARS. For more information, please contactSTARS@ucf.edu.

\section{Recommended Citation}

Hudson, Darren D.; Kutz, J. Nathan; Schibli, Thomas R.; Chao, Qing; Christodoulides, Demetrios N.; Morandotti, Roberto; and Cundiff, Steven T., "Fixed-point attractor for chirp in nonlinear waveguide arrays" (2012). Faculty Bibliography 2010s. 2772.

https://stars.library.ucf.edu/facultybib2010/2772

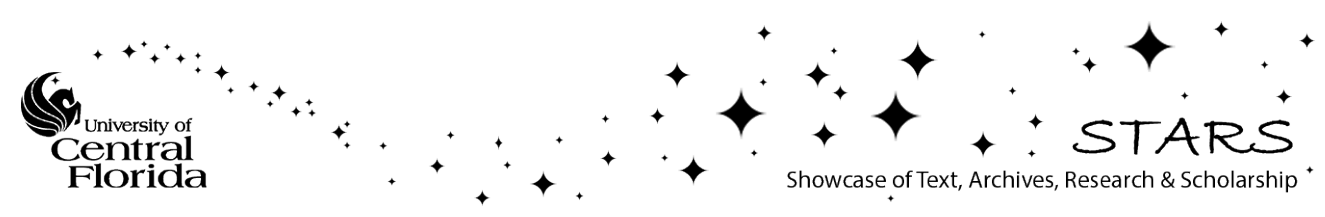




\section{Authors}

Darren D. Hudson, J. Nathan Kutz, Thomas R. Schibli, Qing Chao, Demetrios N. Christodoulides, Roberto Morandotti, and Steven T. Cundiff 


\title{
Fixed-point attractor for chirp in nonlinear waveguide arrays
}

\author{
Darren D. Hudson, ${ }^{1,2, *}$ J. Nathan Kutz, ${ }^{3}$ Thomas R. Schibli, ${ }^{1,2}$ Qing Chao, ${ }^{1,4}$ Demetrios N. Christodoulides, ${ }^{5}$ \\ Roberto Morandotti ${ }^{6}{ }^{\text {and Steven T. Cundiff }}{ }^{1,2,4, \dagger}$ \\ ${ }^{1}$ JILA, National Institute of Standards and Technology and University of Colorado, Boulder, Colorado 80309-0440, USA \\ ${ }^{2}$ Department of Physics, University of Colorado, Boulder, Colorado 80309-0390, USA \\ ${ }^{3}$ Department of Applied Mathematics, University of Washington, Seattle, Washington 98195-2420 \\ ${ }^{4}$ Department of Electrical, Computer and Energy Engineering, University of Colorado, Boulder, Colorado 80309-0425, USA \\ ${ }^{5}$ College of Optics and Photonics, CREOL, University of Central Florida, Orlando, Florida 32816 \\ ${ }^{6}$ Institut National de la Recherche Scientifique, Université du Quebec, Varennes, Canada
}

(Received 15 October 2010; revised manuscript received 13 December 2011; published 30 March 2012)

\begin{abstract}
The propagation of ultrashort optical pulses in an AlGaAs waveguide array is studied using frequency-resolved optical gating measurements. In the nonlinear regime, the measurements show that the pulses at the output of the array evolve toward a set chirp value that is independent of the input chirp. Simulations reproduce the experimental results. The observations can be described as a fixed-point attractor on a chirp-intensity map.
\end{abstract}

DOI: 10.1103/PhysRevA.85.031806

PACS number(s): 42.65.Wi, 42.65.Hw, 42.65.Re

Discrete solitons represent self-trapped states in nonlinear periodic structures that result from the interplay between discrete diffraction and nonlinearity [1,2]. This family of self-localized entities was first observed by Eisenberg et al. in 1998 [3] and has continued to attract substantial attention [2,4-9]. In general, optical discrete solitons can be excited in a variety of physical systems having different types of nonlinearity. Even though most experiments in waveguide lattices have been carried out using pulses, temporal effects in such nonlinear periodic environments have so far remained largely unexplored. More recently, measurement of the temporal profile at the output of waveguide arrays has led to the observation of light bullets [10], $\mathrm{X}$ waves [11,12], and nonlinear pulse shortening [13]. These measurements were based on intensity autocorrelations or cross-correlations and thus were not sensitive to the phase dynamics of the output pulse.

In this Rapid Communication, we investigate the impact of a waveguide array's nonlinear dynamics on the full electric field of an ultrashort pulse as a function of both input chirp and average power. Surprisingly, we find that, at sufficiently high peak power, the waveguide array sets the output pulse's chirp to a fixed value, regardless of the chirp on the input pulse. This behavior is reproduced in simulations and can be classified as a fixed-point attractor in a phase space involving intensity and chirp. Attraction effects have manifested in dissipative nonlinear optical systems through the formation of similaritons [14]. However, previous theory and experiments have studied similaritons in a single dimension, that is, temporal or spatial, whereas our observations correspond to the formation of a self-similar structure in both space and time. From an applied perspective, these results provide an effective method for shortening and dechirping a pulse without needing detailed

\footnotetext{
*Present address: Institute of Photonics and Optical Science and the Centre for Ultrahigh bandwidth Devices for Optical Systems, CUDOS, School of Physics, University of Sydney, Camperdown 2006, Australia.

†cundiffs@jila.colorado.edu
}

knowledge of the input pulse. Such a method would be useful in telecommunications and for pulse cleanup in amplifier chains. Furthermore, a waveguide array could be used as a robust mode-locking element in a laser [15].

The dynamic exchange of electromagnetic energy in waveguiding systems has long been theoretically considered in the context of coupled-mode theory [16]. Coupled-mode theory provides an ideal and highly simplified analytic framework describing the coupling and propagation of electromagnetic energy in waveguides and waveguide arrays, even when subject to the Kerr nonlinearity [1]. The theory assumes that the electromagnetic field is localized transversely in each waveguide and that the exchange of energy between waveguides can be accurately modeled by an evanescent, linear coupling determined by an overlap integral between waveguide modes [16]. The nonlinear theory, which includes selfphase modulation, has been demonstrated to agree remarkably well with experiments using both $\mathrm{cw}[17,18]$ and pulsed femtosecond [13] light. In the waveguide array configuration considered here, nearest-neighbor interactions dominate the waveguide array dynamics and linear loss is insignificant. This limit leads to a set of discretely coupled nonlinear Schrödinger equations governing the evolution of electromagnetic energy,

$$
\begin{aligned}
& i \frac{\partial A_{n}}{\partial z}-\frac{\beta^{\prime \prime}}{2} \frac{\partial^{2} A_{n}}{\partial t^{2}}+\gamma\left|A_{n}\right|^{2} A_{n}+c\left(A_{n+1}+A_{n-1}\right) \\
& \quad+i \sigma\left|A_{n}\right|^{4} A_{n}=0,
\end{aligned}
$$

where $A_{n}$ represents the normalized electric-field amplitude in the $n$th waveguide $(n=-N, \ldots,-1,0,1, \ldots, N$ and there are $2 N+1$ waveguides). The linear coupling coefficient is $c$ and the nonlinear self-phase modulation parameter is $\gamma$. Unlike previous cw experiments $[1,17,18]$, the femtosecond pulse propagation considered here requires that we explicitly consider chromatic dispersion, which is described by the parameter $\beta^{\prime \prime}$. Furthermore, the effects of threephoton absorption is modeled by the quintic loss term with coefficient $\sigma$.

To experimentally determine the phase dynamics imposed on an ultrashort optical pulse due to nonlinear propagation in a waveguide array, we use frequency-resolved optical gating 


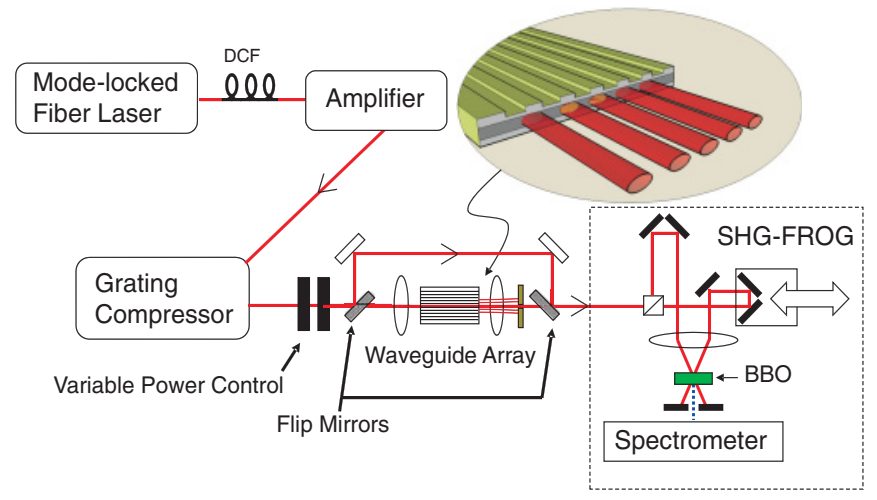

FIG. 1. (Color online) Experimental setup. The output of the fiber laser is broadened by dispersion compensating fiber (DCF) to avoid nonlinearities in the amplifier. The diffraction grating compressor allows tuning of the chirp for the input pulses. The variable power control consists of a half-wave plate and a polarizer. The flip mirrors allow for characterization of the pulses before they enter the waveguide array. Light in the central waveguide is apertured and sent to a background-free SHG-FROG apparatus.

(FROG) to measure the full electric field of pulses output by a waveguide array. A mode-locked, erbium-doped fiber laser with a repetition rate of $25 \mathrm{MHz}$ (operating at $1550 \mathrm{~nm}$ ) and a chirped-pulse amplifier and compressor system are used to generate intense input pulses (see Fig. 1). Using dispersion compensating fiber (DCF), the normally chirped pulses from the fiber laser are further stretched to several picoseconds to avoid nonlinearities in the amplifier. These stretched pulses are coupled to a bidirectionally pumped erbium amplifier [19], which increases the pulse energy by a factor of 7 , while maintaining their original pulse shapes. The output of the amplifier is temporally compressed or stretched in free space by a diffraction grating compressor. This system produces pulse energies of $3.5 \mathrm{~nJ}$ and allows us to change the pulse chirp from normal $\left(+200000 \mathrm{fs}^{2}\right)$ to anomalous $\left(-100000 \mathrm{fs}^{2}\right)$. The pulse spectrum is centered at $1550 \mathrm{~nm}$ with a width of $40 \mathrm{~nm}$ at the $-10 \mathrm{~dB}$ points, although there is significant structure, resulting in a Fourier transform limited duration of approximately $220 \mathrm{fs}$.

The pulses are coupled into the waveguide array using standard microscope objectives $(40 \times)$ mounted on three-axis stages. The input field is mode matched to the waveguide with a coupling efficiency $>40 \%$. The 6 -mm-long waveguide array has a $10-\mu \mathrm{m}$ center-to-center spacing between waveguides, with $1.5 \mu \mathrm{m}$ tall ridges and $4 \mu \mathrm{m}$ wide waveguides. Index guiding in the vertical direction is provided by a core layer consisting of $\mathrm{Al}_{0.18} \mathrm{Ga}_{0.82} \mathrm{As}$ and cladding layers consisting of $\mathrm{Al}_{0.24} \mathrm{Ga}_{0.76} \mathrm{As}$. All results presented in here are collected in the nonlinear regime such that discrete spatial solitons form, which at high-enough intensity clamp in their spatial distribution due to three-photon absorption [20].

To measure the electric fields, we use FROG based on second harmonic generation [21]. This method involves recording a spectrally resolved autocorrelation or spectrogram. We use a computer-controlled delay stage in conjunction with a spectrometer to acquire the spectrogram. The information contained in the spectrogram is sufficient to retrieve the full pulse shape and phase using an iterative algorithm [21].

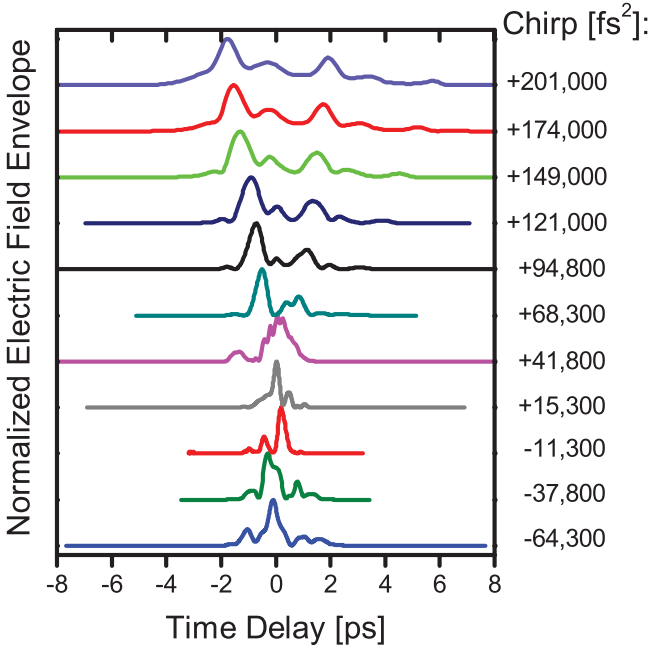

FIG. 2. (Color online) Electric-field envelopes before the waveguide as a function of time and compressor setting. The plot shows the electric fields as the compressor is tuned from the most normal dispersion setting (top trace) through the zero group delay dispersion (GDD) point (near the fourth trace from bottom) to the most anomalous setting (bottom trace).

FROG has several advantages over autocorrelation. A FROG measurement gives the full electric field and thus reveals asymmetry in the electric-field envelope. Second, the intensity autocorrelation is insensitive to the phase dynamics of the electric field. The inversion algorithm for FROG, however, calculates the spectral (or temporal) phase based on the spectrogram.

Using FROG, we mapped out the electric fields before and after the waveguide array. By tuning the distance between diffraction gratings in the compressor, the linear frequency chirp of the input pulse is varied smoothly over a wide range. Increasing the chirp stretches the pulse in the time domain as shown in Fig. 2. By fitting the measured phase evolution to a polynomial function, we can extract the quadratic term, which corresponds to linear frequency chirp. The variation in chirp matched well to the value estimated from the compressor geometry. We coupled each of these fields (at the highest average power available) into the waveguide array and measured the field that emerged from the central waveguide. The resulting electric-field envelopes are shown in Fig. 3. The effect of the waveguide array is clear: It shortens the pulses in the time domain such that the duration of the output pulse is roughly independent of the input pulse duration. This behavior was first observed via intensity autocorrelations for one input chirp [13]. As Fig. 3 shows, this electric-field reshaping occurs over a wide range of input chirps.

In addition to shortening the pulse, the waveguide array also clamps its chirp to a fixed value, as shown in Fig. 4(a), independent of the input pulse. Simple material dispersion will add a constant offset to the input chirp, as shown in the figure, but does not result in a constant output chirp. Thus, we attribute this effect to nonlinearity in the waveguide. To verify that the chirp clamping is a nonlinear phenomenon, we repeated the measurement at several power levels and observe that it disappears at the lowest power level. At low power, the output chirp tracks the input pulse. To further investigate 


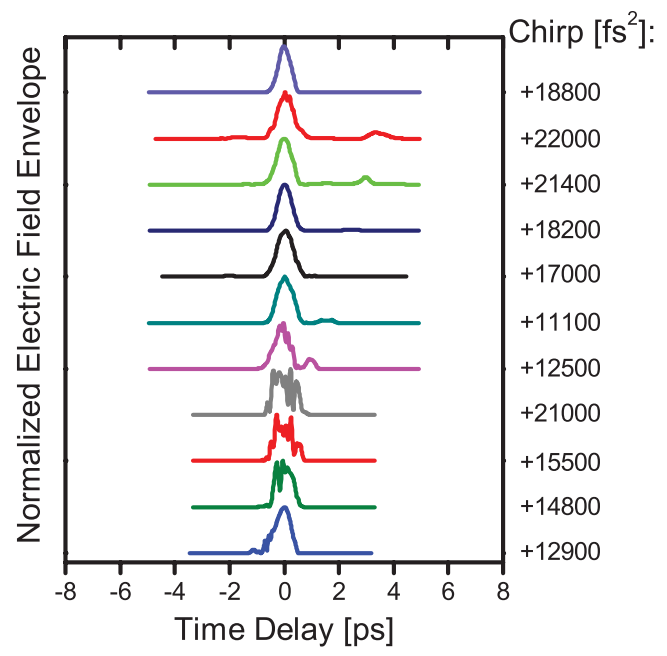

FIG. 3. (Color online) Electric-field envelopes after the waveguide as a function of time and compressor setting. Each trace corresponds, in the same ordering, to an input shown in Fig. 2. The rapid oscillations near the zero dispersion point are due to self-phase modulation in the material.

and understand this phenomena, we also performed numerical simulations for conditions matching the experiments.

Simulations shown in Fig. 4(b) of the mode-coupling dynamics, as given by the governing equation (1), reproduce the experimental results. The simulations are performed with a pseudospectral method that spectrally transforms the timedomain solution and uses a fourth-order Runge-Kutta for propagation in the waveguide. For all simulations, a total of $41(N=20)$ waveguides are considered to match the waveguide array geometry and design. The linear coupling coefficient and dispersion are determined experimentally to be $c=0.82 \mathrm{~mm}^{-1}$ and $\beta^{\prime \prime}=+1500 \mathrm{fs}^{2} / \mathrm{mm}$, respectively, and the nonlinear self-phase modulation parameter of $\gamma=$ $3.6 \mathrm{~m}^{-1} \mathrm{~W}^{-1}$ is used $[17,18]$. The simulations use the same waveguide length as the experiments.

To study the effects of chirp on femtosecond pulses, simulations are performed for a chirped hyperbolic secant pulse launched into the center waveguide $(n=0)$. Thus, the initial condition for (1) is

$$
A_{0}(0, t)=\eta \operatorname{sech}(\omega t) \exp \left(i \alpha t^{2}\right),
$$

where $\alpha$ determines the amount of normal $(\alpha>0)$ or anomalous $(\alpha<0)$ linear chirp. The pulse amplitude $(\eta)$ and width $(\omega)$ parameters are chosen so as to generate pulses with peak powers matching our experiments. The simulation results of (1) with the launch condition (2) are critical in confirming our claims.

The experimental and simulation results show similar behavior. The lowest average power level (in the central waveguide) of $11 \mathrm{~mW}$ corresponds to the threshold of the self-focusing regime for this waveguide array. The reshaping vanishes at this power, and the output chirp of the pulse in the central waveguide closely follows that of the input chirp. At the medium power level $(18 \mathrm{~mW})$, reshaping is observed for input chirps less than $+130000 \mathrm{fs}^{2}$. At these chirp levels, the pulse is too stretched out in time to achieve the necessary peak power to undergo full reshaping. Thus, the outlier points

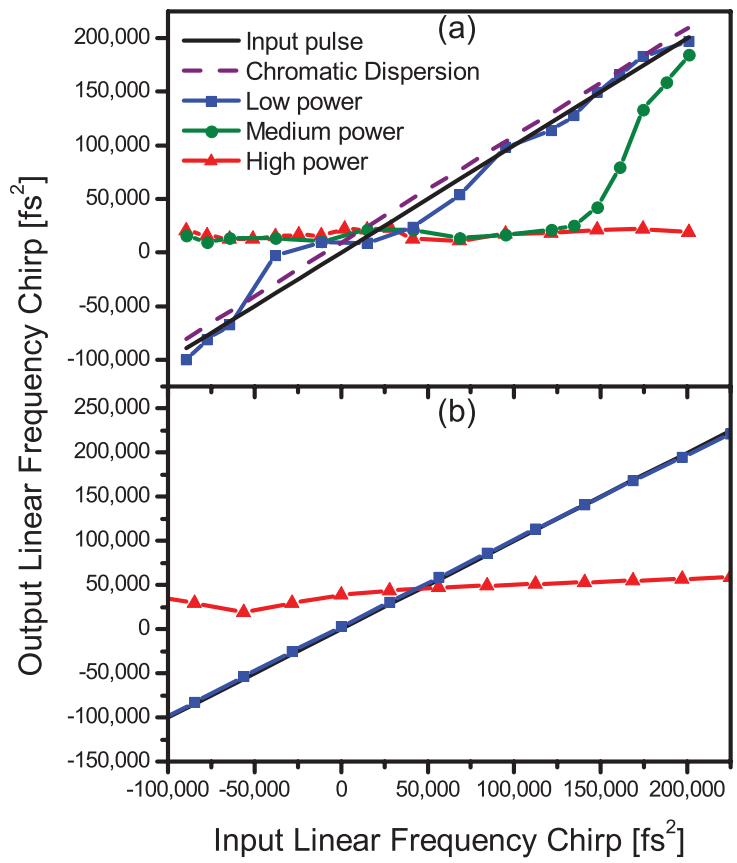

FIG. 4. (Color online) (a) Experimentally measured chirp of the input pulses and output pulses at three average power levels: low $(11 \mathrm{~mW})$, medium $(18 \mathrm{~mW})$, and high $(24 \mathrm{~mW})$. The abscissa of this plot was calibrated by measuring the chirp on the input pulses from the compressor as a function of distance between gratings. For comparison, the solid black line indicates the input chirp and the dashed line shows the expected output chirp due to dispersion alone. (b) Numerical results of the output chirp of the waveguide array corresponding to low- and high-power experimental cases. The simulation agrees well with the experimental results.

rapidly move toward the input chirp from the compressor. At the highest average power $(24 \mathrm{~mW})$, the waveguide array sets the output GDD for all inputs to around $+17000 \mathrm{fs}^{2}$, with a standard deviation of $3800 \mathrm{fs}^{2}$. Particularly interesting is that the waveguide array does this for both normal and anomalous input chirp. One slight difference between the numerics and the experiment is that the simulation shows a slightly higher fixed value of chirp for the output pulses than the experimental value, which we attribute to approximations made in the model and uncertainty in experimental parameters. The parameters used in the simulation were not adjusted to improve the agreement with experiment.

We can classify the effect of the waveguide array as a fixed-point attractor in a parameter space of output chirp and output power. As the average power of the input pulses is increased, the attraction toward an average fixed point of an output power of $4.55 \mathrm{~mW}$ and a chirp of $+16600 \mathrm{fs}^{2}$ becomes stronger. Figure 5 helps to visualize this idea for the experimental results by plotting vectors in a (chirp,power) plane. For each input condition a vector is plotted with its tail at the input locus and pointing toward the output locus. The length of the vector is proportional to the distance between the input and the output loci. The location of the fixed-point attractor is presented as well, with error bars showing the standard deviation of the output values in both coordinates. Clearly for high power, all vectors point toward the same fixed point. For medium power, most of them do, with the exception 


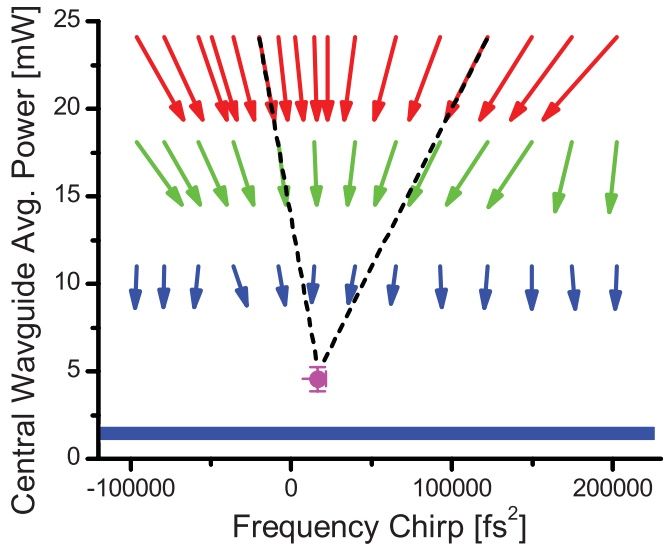

FIG. 5. (Color online) A vector plot showing the fixed-point attractor in the (chirp,power) plane for the experimental results. The attractor is denoted by a square. The upper arrows (red) are for $24 \mathrm{~mW}$, the middle arrows (green) are for $18 \mathrm{~mW}$, and the lowest arrows (blue) are for $11 \mathrm{~mW}$. The dashed lines are to guide the eye. For the lowest power, there is no attractor and the output loci fall in the blue region.

of those with the highest chirp. At the lowest power, none of them do. At low power, the output loci fall in the blue band. We have done experiments on a second sample that has several waveguide arrays with varying spacing between waveguides. While this sample was fabricated to be the same as the original sample, there are unavoidable differences in parameters such as the alloy concentrations, length, etch depth, etc. This sample also exhibits a fixed-point attractor, although the position is different, and clearly depends on coupling strength. These results demonstrate that the presence of a fixed-point attractor is a robust phenomenon and not specific to the sample parameters.

We have run the simulations without three-photon absorption $(\sigma=0)$ and for a single waveguide rather than an array. In both cases, the fixed-point attractor is not present. Thus, we conclude that the presence of both nonlinearity and discrete diffraction play essential roles in our observations. We also varied the simulation parameters and found that the presence of a fixed-point attractor persists over a reasonably large volume of parameter space; hence, it is a robust effect, although its position depends on the parameters.

The output pulse clearly has solitonlike features in the sense that a wide range of input conditions evolve toward the same output state. However, our results cannot be due to solitonic effects in the time domain. The array dispersion is normal, which forbids the formation of temporal solitons. This conclusion is also supported by the fact that the output chirp is normal. Rather we attribute these observations to a balance between dispersion and temporal reshaping due the formation of a discrete spatial soliton for the central part of the pulse, while the wings are shed into the other waveguides. Varying the parameters in the simulations show that both three-photon absorption and coupling to adjacent waveguides are essential to our observations. Both of these are dissipative processes. Further insight into the existence of a fixed-point attractor might be gained by considering a single waveguide and treating the coupling to the rest of the array as a dissipative process. This approximation would correspond to a dissipative temporal soliton [22].

In summary, we have shown experimentally that, in the nonlinear regime, the chirp and power of an ultrashort pulse can become independent of the input chirp and power after propagation through a waveguide array. The observed dynamics are in good agreement with numerical simulations. We believe that these results represent a new temporal-spatial paradigm to nonlinear propagation in discrete systems. While similar to temporal solitons, it is fundamentally different because it occurs in the normal dispersion regime. We believe that our observations are the time-domain analog to self-accelerating, self-trapped optical beams, which have recently been described [23].
[1] D. N. Christodoulides and R. I. Joseph, Opt. Lett. 13, 794 (1988).

[2] F. Lederer, G. I. Stegeman, D. N. Christodoulides, G. Assanto, M. Segev, and Y. Silberberg, Phys. Rep. 463, 1 (2008).

[3] H. S. Eisenberg, Y. Silberberg, R. Morandotti, A. R. Boyd, and J. S. Aitchison, Phys. Rev. Lett. 81, 3383 (1998).

[4] R. Iwanow, R. Schiek, G. I. Stegeman, T. Pertsch, F. Lederer, Y. Min, and W. Sohler, Phys. Rev. Lett. 93, 113902 (2004).

[5] J. Fleischer, G. Bartal, O. Cohen, T. Schwartz, O. Manela, B. Freedman, M. Segev, H. Buljan, and N. Efremidis, Opt. Express 13, 1780 (2005).

[6] A. Szameit, J. Burghoff, T. Pertsch, S. Nolte, A. Tuennermann, and F. Lederer, Opt. Express 14, 6055 (2006).

[7] D. N. Neshev, A. A. Sukhorukov, A. Dreischuh, R. Fischer, S. Ha, J. Bolger, L. Bui, W. Krolikowski, B. J. Eggleton, A. Mitchell, M. W. Austin, and Y. S. Kivshar, Phys. Rev. Lett. 99, 123901 (2007).

[8] R. Dong, C. E. Rüter, D. Kip, O. Manela, M. Segev, C. L. Yang, and J. J. Xu, Phys. Rev. Lett. 101, 183903 (2008).
[9] Y. V. Kartashov, V. A. Vysloukh, and L. Torner, Prog. Opt. 52, 63 (2009).

[10] D. Cheskis, S. Bar-Ad, R. Morandotti, J. S. Aitchison, H. S. Eisenberg, Y. Silberberg, and D. Ross, Phys. Rev. Lett. 91, 223901 (2003).

[11] Y. Lahini, E. Frumker, Y. Silberberg, S. Droulias, K. Hizanidis, R. Morandotti, and D. N. Christodoulides, Phys. Rev. Lett. 98, 023901 (2007).

[12] S. Droulias, K. Hizanidis, J. Meier, and D. N. Christodoulides, Opt. Express 13, 1827 (2005).

[13] D. Hudson, K. Shish, T. Schibli, J. Kutz, D. Christodoulides, R. Morandotti, and S. Cundiff, Opt. Lett. 33, 1440 (2008).

[14] J. M. Dudley, C. Finot, D. J. Richardson, and G. Millot, Nat. Phys. 3, 597 (2007).

[15] J. Proctor and J. Kutz, Opt. Lett. 30, 2013 (2005).

[16] D. Marcuse, Theory of Dielectric Optical Waveguides (Academic Press, New York, 1974). 
[17] H. S. Eisenberg, R. Morandotti, Y. Silberberg, J. M. Arnold, G. Pennelli, and J. S. Aitchison, J. Opt. Soc. Am. B 19, 2938 (2002).

[18] U. Peschel, R. Morandotti, J. M. Arnold, J. S. Aitchison, H. S. Eisenberg, Y. Silberberg, T. Pertsch, and F. Lederer, J. Opt. Soc. Am. B 19, 2637 (2002).

[19] F. Tauser, A. Leitenstorfer, and W. Zinth, Opt. Express 11, 594 (2003).
[20] D. D. Hudson, J. N. Kutz, T. R. Schibli, D. N. Christodoulides, R. Morandotti, and S. T. Cundiff, Opt. Express 20, 1939 (2012).

[21] D. Kane and R. Trebino, IEEE J. Quantum Electron. 29, 571 (1993).

[22] N. N. Akhmediev and A. Ankiewicz (eds.), Dissipative Solitons (Springer-Verlag, Berlin, 2005).

[23] I. Kaminer, M. Segev, and D. N. Christodoulides, Phys. Rev. Lett. 106, 213903 (2011). 